\title{
EL PROFESOR UNIVERSITARIO: \\ CONSTRUCCIÓN DE SU SABER PEDAGÓGICO \\ E IDENTIDAD PROFESIONAL A PARTIR DE SUS \\ COGNICIONES Y CREENCIAS
}

\section{Introducción}

Uno de los grandes desafíos de esta investigación ha sido, sin duda, lograr el acercamiento al docente universitario y su trabajo en el aula para conocer qué piensan, perciben o creen los profesores respecto del proceso enseñanza-aprendizaje en la universidad. Su gran desafío es encontrar significado a su propia experiencia pedagógica.

El profesor está continuamente intentando aprender más sobre sí mismo y cómo mejorar las interacciones en el aula. Algunos autores han caracterizado la construcción del conocimiento en la enseñanza como "un vuelco paradigmático", al señalar que es tiempo de reconocer la esterilidad de un conocimiento científico y tecnológico que, por sí solo, guíe la actuación de los profesionales. Esto reproduciría la dicotomía entre el conocimiento y la actuación en la práctica cotidiana (Biddle, Good y Goodson, 2000; Camps, 2001; Cárdenas, Rodríguez y Torres, 2000; Crookes, 2003).

La investigación acerca de los pensamientos y conocimientos de los profesores/as tiene como propósito comprender las concepciones, creencias, dilemas y teorías que gobiernan la práctica profesional, cómo identificar los procesos que constituyen el "aprender a enseñar" y las categorías conceptuales en las que se articula el conocimiento para desarrollar la actividad profesional de la enseñanza.

Este estudio tiene como objetivo abordar las creencias que los académicos no pedagogos sostienen para interpretar la multitud de variables que ocurren en el fenómeno de la transposición didáctica, entendida ésta como el proceso de hacer enseñable el conocimiento disciplinar. 


\section{Marco teórico}

El problema de esta investigación se sitúa en el campo de los procesos de enseñanza-aprendizaje universitarios. La idea de explorar el fenómeno didáctico desde las creencias de los docentes no pedagogos se relaciona con la alta cuota de subjetividad y complejidad que dicho fenómeno reviste. Por otro lado, la actuación del docente y su concepción del proceso de enseñanza-aprendizaje se comprende al entrar al entramado de creencias que sostiene su praxis pedagógica.

Las creencias ponen de manifiesto la existencia de procesos particulares de construcción del conocimiento de los profesores, a partir de los cuales interpretan, deciden e intervienen en las situaciones de enseñanza-aprendizaje. Las creencias sirven como marco de referencia y guías "orientadoras" de la acción en la práctica educativa.

\subsection{Los estudios sobre el pensamiento del profesorado}

Una de las líneas de investigación que más se ha desarrollado en las últimas décadas en torno al conocimiento profesional es sin duda la del "pensamiento del profesor", hasta el punto de que se habla incluso del "paradigma del pensamiento del profesor". Su interés fundamental es conocer los procesos de razonamiento que ocurren en la mente del docente durante su actividad profesional.

A comienzos de los 70 -durante el auge de la Psicología Cognitiva- aparecen nuevos enfoques, como los estudios sobre el conocimiento práctico del docente. Esta línea de trabajo surge para paliar algunas de las carencias de los enfoques de procesamiento de información; es decir, ante el reduccionismo, la excesiva formalización y la descontextualización, emergen aproximaciones prácticas e integradoras que postulan que el conocimiento que facilita la comprensión del contexto de actuación docente y que determina en última instancia las decisiones y cursos de acción durante la enseñanza, es un conocimiento personal o práctico-reflexivo, producto de la biografía y experiencia del docente, de sus conocimientos actuales y de su relación activa con la práctica. Es un "saber hacer" en su mayor parte tácito, que se activa en la acción misma (Frabboni, 
2001; Gelhard y Oprandy, 1999; González, Río y Rosales, 2001; Johnson y Golombek, 2002).

Los profesores afrontan su actividad profesional mediante un sistema idiosincrásico de conocimientos, producto de la elaboración personal de sus ideas en un contexto institucional y social determinado. Dichos conocimientos son los que efectivamente utiliza el docente y en "su conformación entran en juego factores subjetivos, biográficos y experienciales, así como aspectos objetivos contextuales" (Kansanen, Tirri, Meri, Krokfors, Huso y Jyrhämä, 2000; Libedinsky, 2001; Menin, 2001; Muchmore, 2004).

El docente es, sin duda, un sujeto que continuamente construye, elabora y prueba su teoría personal del mundo. Esto es consistente con la visión actual de la enseñanza, considerada como una actividad del pensamiento profesional, en la que el cambio conceptual debe ser reconocido como el centro del aprendizaje del docente. Las teorías personales se definen como un sistema en el que subyacen constructos que el docente usa cuando piensa, evalúa, clasifica y guía su práctica pedagógica (Richard-Amato, 2003; Sanjurjo, 2002; Tsui, 2003; Undurraga, 2007; Woods, 1996). Ellas responden casi siempre al sentido común y son de naturaleza tácita. No son necesariamente coherentes; sino, más bien, se consideran como dinámicas y sujetas al cambio y a la reformulación gradual.

\section{2. ¿Qué son las creencias?}

Las personas utilizan las creencias para recordar, interpretar, predecir y controlar los sucesos que ocurren y tomar decisiones. Son producto de la construcción del mundo y se basan en procesos de aprendizaje asociativo; pero también tienen un origen cultural, en tanto se construyen en "formatos de interacción social y comunicativa" (Goodson y Numan, 2002; Hamel, 2003; Hashweh, 2005; Johnstone, 2000; Sevillano; 2007). Las creencias están compuestas por conjuntos más o menos integrados y consistentes de ideas que se construyen a partir de las experiencias cotidianas. Son versiones incompletas y simplificadas de la realidad que, si bien permanecen inaccesibles a la conciencia, tienen algún nivel de organización interna, estructuración y sistematicidad. 
Las creencias del profesor están relacionadas con situaciones especificas orientadas a la actuación e incluyen tanto las creencias sobre su trabajo (objetivos, concepciones de sus estudiantes, contenidos, entre otras) como las "ways in which they [give] meaning to these beliefs by their behavior in the classroom" (Andrews, 2003; Ben-Peretz, 2002; Carusetta y Cranton, 2005; Freeman, 2002).

Borg (2003) usa el término "cognición del docente" para referirse a las dimensiones cognitivas no observables de la enseñanza, es decir, lo que los docentes conocen, creen, y piensan. Ellos son agentes activos en la toma de decisiones instruccionales haciendo uso de complejas redes de conocimientos, pensamientos y creencias. Estas redes se orientan a la práctica, son personalizadas y sensibles al contexto.

\subsection{La identidad profesional}

La identidad profesional se refiere a una entidad individual que el profesor construye a partir de su relación con su espacio de trabajo y grupo profesional de referencia. Conlleva un fenómeno de apropiación de modelos que provienen de las políticas sociales y opciones políticas de una sociedad. En este sentido, los estudios acerca de la identidad profesional se refieren a un grupo de sujetos situados en un contexto socioeducativo específico.

El concepto de identidad tiene un carácter dinámico y de transformación constante, que evidencia una permanencia y estabilidad en el tiempo, y es producto de la interacción con otros en un proceso de continua construcción o reconstrucción. En este proceso el docente desarrolla las creencias pedagógicas que guían su actuación en el aula y responden al contexto socioeducativo en el que se inserta (González, Río y Rosales, 2001).

La identidad profesional se consolida cuando cada profesor descubre sus concepciones básicas acerca del proceso didáctico y emergen sus creencias implícitas acerca de sí mismo y de los otros, sean éstos colegas o estudiantes. En definitiva, las concepciones y autopercepciones que construyen los docentes en torno a su labor pedagógica son esenciales para lograr su identidad, tanto personal como profesional (Borg, 2003). 


\section{Diseño de investigación}

\subsection{Pregunta de investigación}

¿Cuáles son las creencias que los académicos universitarios de Trabajo Social y Periodismo de dos universidades chilenas tienen acerca del proceso enseñanza-aprendizaje?

\subsection{Supuestos de esta investigación}

- El docente es un sujeto reflexivo que experimenta situaciones de enseñanza-aprendizaje y les otorga significado.

- Un profesor informado debería tener una base de conocimiento más profunda acerca del proceso enseñanza-aprendizaje.

- La reflexión lleva a la toma de decisiones.

- El autoconocimiento respecto del proceso enseñanza-aprendizaje se logra mediante la revisión crítica y reflexiva de las prácticas pedagógicas.

\subsection{Justificación de los supuestos}

El profesor es un sujeto reflexivo, racional, que toma decisiones, emite juicios, tiene creencias y genera rutinas propias de su desarrollo profesional. Además, las creencias del docente guían y orientan su conducta. Los docentes no están frecuentemente conscientes del tipo de enseñanza que realizan o cómo ellos manejan muchas decisiones emergentes en el aula. Puesto que muchas cosas suceden simultáneamente durante una clase, es algunas veces difícil, para ciertos docentes, estar conscientes de lo que allí sucede.

\subsection{Objetivo general}

Comprender las creencias de los docentes no pedagogos en torno al funcionamiento de las variables didácticas que intervienen en el proceso enseñanza-aprendizaje en contextos universitarios.

\subsection{Metodología}

La presente investigación corresponde a un estudio de carácter descriptivo-interpretativo, congruente con un diseño de estudio de 
casos múltiples, puesto que indaga en las creencias de los docentes universitarios en el contexto del proceso enseñanza-aprendizaje. El caso objeto de análisis de este estudio está constituido por un grupo de académicos provenientes de las carreras de Periodismo y Trabajo Social de dos universidades chilenas, que han sido agrupados en torno del área de Ciencias Sociales, puesto que la pertenencia a dicha área del conocimiento incide en el tipo de creencias y actuaciones pedagógicas que demuestran dichos docentes, tanto en su discurso como en su desempeño en el aula. La literatura de metodología de investigación la identifica como un estudio intrínseco de caso, es decir, "el estudio se lleva a cabo porque se desea alcanzar una mayor comprensión de los casos en particular. No se han seleccionado los casos porque representen a otros o porque representen un rasgo o problema particular" (Sandín, 2003).

Se pretende realizar un análisis de la realidad socioeducativa mediante un proceso de indagación que se caracteriza por el examen detallado, comprehensivo, sistemático y en profundidad del caso objeto de estudio.

\subsection{Sujetos informantes}

Los académicos no pedagogos que participan en este estudio imparten docencia en dos universidades chilenas pertenecientes al Consejo de Rectores (CRUCH). Estos 35 profesores provienen de diversas áreas del conocimiento. Para propósitos de este artículo se trabajará con profesores de las carreras de Periodismo y Trabajo Social.

\subsection{Técnica de recopilación de información}

3.7.1. Entrevista semiestructurada aplicada a cada uno de los sujetos informantes, con el fin de desvelar sus creencias respecto del proceso enseñanza-aprendizaje en contextos universitarios.

3.7.2. Narración autobiográfica consistente en una actividad de reflexión individual y escrita, en la cual los informantes debían indicar aquellos hitos académicos y profesionales relevantes respecto de su formación como docentes. Este instrumento permitió la triangulación de la información entregada por la entrevista semiestructurada. En 
los últimos tiempos, la investigación en educación ha considerado cada vez más la autorreflexión como una poderosa herramienta para obtener información de los docentes. Ésta se puede realizar mediante diarios autobiográficos o narraciones específicas, en las cuales los profesores consignan un registro de sus reflexiones. Richards y Nunan (1990) señalan que los diarios autobiográficos y las actividades de autorreflexión reflejan las creencias y los supuestos en que se basan las prácticas docentes. Además, este ejercicio permite al profesor estar consciente de su actuar pedagógico y generar buenos hábitos en sus prácticas.

\subsection{Procedimiento de análisis de los datos}

La discusión bibliográfica permitió establecer a priori las categorías del estudio. Éstas se condicen fundamentalmente con las variables didácticas del proceso enseñanza-aprendizaje. Una vez que los datos de ambos instrumentos fueron recogidos y transcritos durante el primer y segundo semestre de 2007, se realizó un análisis de contenido y comparación de las unidades de información, con el fin de codificarlas. Estas unidades fueron sometidas a la triangulación por investigador y por método investigativo hasta que se produjo la saturación de los datos. Posteriormente, se establecieron patrones recurrentes que permitieron describir los datos, para luego descubrir los significados e interpretaciones que los propios informantes otorgaban a sus creencias pedagógicas.

\subsection{Análisis de los datos}

Los datos fueron organizados en dos dimensiones que describen las creencias de los informantes respecto de las variables didácticas del proceso enseñanza-aprendizaje. La primera dimensión cuenta con dos categorías y la segunda con siete. A continuación se muestra la organización de los datos: 


\begin{tabular}{|l|l|}
\hline DIMENSIÓN & CATEGORÍAS \\
\hline La profesionalización docente & $\begin{array}{l}\text { Creencias sobre las etapas claves en la formación y } \\
\text { desarrollo como docente }\end{array}$ \\
\hline & $\begin{array}{l}\text { Creencias respecto de las mejores experiencias pedagógicas } \\
\text { de los informantes y su impacto en sus prácticas docentes }\end{array}$ \\
\hline El proceso enseñanza-aprendizaje & El estudiante de educación superior \\
\hline & El proceso de aprendizaje \\
\hline & Las características de un buen docente \\
\hline & El plan de estudios y los contenidos de enseñanza \\
\hline & La metodología de enseñanza \\
\hline & La evaluación de los aprendizajes \\
\hline & $\begin{array}{l}\text { La actuación docente, el discurso y el impacto que provoca } \\
\text { en los estudiantes }\end{array}$ \\
\hline
\end{tabular}

\section{Resultados del estudio}

\subsection{Dimensión de la profesionalización docente}

4.1.1. Categoría: creencias sobre las etapas claves en la formación y desarrollo como docente

Respecto de esta categoría, los sujetos informantes declaran que una de las etapas más importante en su desarrollo como docentes fue su formación de pregrado, ya que tuvieron oportunidad de realizar ayudantías y luego desempeñarse como docentes colaboradores en la universidad, así lo demuestra los siguientes fragmentos:

"...una etapa clave en mi formación fue las ayudantías realizadas en mi época de estudiante".

“...una etapa clave en mi formación docente fue cuándo por primera vez asumí como alumna ayudante".

"También me marcó el hecho de haber sido alumno ayudante... además, no era ayudante de cualquier profesor, sino de algunos muy específicos".

Una segunda etapa importante en su formación como docentes fue asumir como profesor part-time y luego ser miembro de la planta docente, y el hecho de asumir como docentes colaboradores. Así lo demuestran las siguientes citas:

"...una segunda etapa importante en mi formación docente fue mi etapa como profesor part-time y mi paso a la planta docente". 
“... la segunda etapa importante en mi formación docente fue cuando asumí como colaboradora, la carga de trabajo aumentó como también mi grado de injerencia en algunas materias y, por supuesto, la autoridad frente a los alumnos".

Como lo ilustran los fragmentos anteriores, el paso a una etapa de trabajo más estable y de mayor responsabilidad marca una gran diferencia en el desarrollo profesional de los entrevistados, puesto que su injerencia y autoridad frente a los estudiantes aumenta cuando asumen la titularidad de las asignaturas que enseñaban como docentes colaboradores.

Algunos de los informantes señalan que es complejo establecer alguna etapa clave en su formación como docentes, pero mencionan que su primera experiencia frente a un grupo de estudiantes marcó profundamente la forma como hoy enfrentan sus funciones docentes, así lo demuestra el siguiente fragmento.

“...la primera experiencia al mando de un grupo de alumnos delineó de manera profunda la manera en que hoy enfrento mi función como profesor..."

Otros entrevistados mencionan como segunda etapa importante en su proceso de formación, sus estudios de posgrado, así lo demuestra la cita siguiente:

"...una etapa clave en mi formación creo que es mi posgrado en España... es decir, hay un antes y después..."

Es importante destacar que para algunos informantes la realización de sus estudios de enseñanza media fue un momento clave en su formación como docentes, puesto que en esa etapa tuvieron como profesores a personas que los marcaron positivamente respecto de la forma cómo enseñan en la universidad. Estos profesores se convirtieron en modelos que guían su actuación pedagógica en la actualidad.

"...lo que uno va aprendiendo de los profesores de enseñanza media es muy importante para uno como formador". 
“...los profesores de enseñanza media son modelos a seguir en mi práctica..."

4.1.2. Categoría: creencias respecto a las mejores experiencias pedagógicas de los informantes y el impacto que éstas han tenido en sus prácticas docentes

En relación con esta categoría, los docentes entrevistados coinciden en que sus mejores experiencias en el ámbito docente están relacionadas con su interacción con los estudiantes.

“...siempre han tenido que ver con mi contacto con los alumnos..."

Además, se incorpora en sus reflexiones el aspecto afectivo y el reconocimiento de los estudiantes como una influencia externa importante que motiva a continuar en la docencia.

“...pienso que en la medida en que los alumnos reconocen el esfuerzo y dedicación de su profesor hay una buena dosis de motivación para seguir esta noble misión..."

En otra de las reflexiones se detallan otras dos experiencias satisfactorias para los informantes, a saber, la oportunidad en que se entregó a los estudiantes el primer certamen corregido y la retroalimentación recibida en la primera evaluación docente, hechos que sirvieron para reafirmar su convicción sobre lo que es hacer docencia. Las relaciones con los estudiantes, la motivación hacia los temas abordados, los resultados logrados y el reconocimiento a la dedicación de los docentes motivan, sin duda, hacia una docencia de calidad.

\subsection{Dimensión del proceso enseñanza-aprendizaje}

\subsubsection{Categoría: el estudiante de educación superior}

Los informantes coincidieron en sus creencias de que los estudiantes de las carreras de Periodismo y Trabajo Social deberían tener habilidades sociales, una buena autoestima y una actitud reflexiva, como lo evidencian los siguientes ejemplos: 
"...habilidades sociales... alta capacidad de comunicación..."

“...una personalidad bastante definida... no puede ser tímido..."

“...buena autoestima, no pueden ser personas con problemas de salud mental..."

“...una persona capaz de realizar un análisis crítico, alguien reflexivo..."

Los informantes de Periodismo consideran importante que el estudiante que ingresa a estudiar esta carrera posea fuertes habilidades comunicativas y seguridad en sí mismo, para así desempeñarse exitosamente y tener un espacio en el mundo laboral.

En el caso de los informantes de Trabajo Social, sus creencias sostienen que el estudiante que estudia esta carrera debe tener una habilidad comunicativa muy desarrollada y, a su vez, debe ser capaz de formular juicios críticos a partir de la reflexión. Según la literatura, esto promueve el desarrollo del pensamiento crítico, ayudando a que los estudiantes desarrollen la capacidad de valorar y mejorar las situaciones que experimentan. El resto de los informantes del área le atribuyen mayor importancia al hecho de que el estudiante no presente ninguna patología psicológica.

\subsubsection{Categoría: el proceso de aprendizaje}

Al preguntar a los sujetos informantes por sus creencias respecto del aprendizaje, queda claro que todos poseen una conceptualización distinta, aunque coinciden en algunos puntos. Hay docentes que coinciden en que el aprendizaje es un proceso de construcción continua a lo largo de la vida como lo indican los siguientes fragmentos:

\footnotetext{
"...como un proceso, es construir conocimientos e instrumentos..."

"...no se acaba, es algo que está en constante construcción..."
} 
"...como un proceso lento, que no puede lograrse de un día para otro..."

Los informantes de Trabajo Social incorporan un nuevo elemento, que se relaciona con poner en práctica lo aprendido, es decir, llevar a la acción el nuevo conocimiento, competencia o actitud aprendida.

"...es cuando tú adquieres un nuevo conocimiento, eres capaz de desarmarlo, manejarlo, trabajarlo y aplicarlo..."

Agrega uno de los informantes que el aprendizaje es un proceso de interacción, un proceso más dialógico, más horizontal entre el que entrega el conocimiento y el que lo recibe.

$\mathrm{Al}$ ser preguntados los informantes sobre sus creencias respecto de cómo aprendían sus estudiantes, ambos grupos, Trabajo Social y Periodismo, declararon situaciones bastante similares, como por ejemplo que sus estudiantes no sabían cómo estudiar y sólo llegaban a un nivel de memorización, como lo evidencian las siguientes respuestas.

"...mis estudiantes estudian mal, estudian de memoria..."

"...mis estudiantes utilizan el...aprendizaje de memoria..."

La mayoría de los informantes declaran que sus estudiantes llegan a la educación terciaria sin hábitos de estudios, sin las competencias básicas requeridas para comenzar su proceso de formación. Esto queda en evidencia al declarar que:

"...mis estudiantes son malos lectores, tienen mala comprensión lectora, se requiere de mucha ejemplificación concreta..."

"...creo que los alumnos no tienen un sistema de aprendizaje, ellos no vienen con un sistema de aprendizaje..." 
Algunos informantes de Periodismo tienen una mirada distinta respecto de cómo aprenden sus estudiantes. La razón es que esta es una carrera con un alto componente práctico y los informantes apunten a desarrollar competencias que sus estudiantes tienen que poner en práctica rápidamente; por esta razón, ellos creen que sus estudiantes aprenden mediante la experiencia, como lo demuestran las siguientes citas tomadas de la entrevista:

"... aprenden mucho por experiencia..."

"...trato de que ellos aprendan haciendo las cosas..."

\subsubsection{Categoría: las características de un buen docente}

En relación con esta categoría, las respuestas del grupo de informantes fueron variadas, pero se logran destacar tres grupos: quienes se centran en el logro satisfactorio de los objetivos de enseñanza; quienes consideran relevante la motivación de parte del docente y la cercanía que éste tenga con sus estudiantes, y quienes, con una visión más holística, consideran el área afectiva y un sólido dominio del conocimiento por parte del docente.

En los fragmentos extraídos de la entrevista se evidencian las creencias de aquellos docentes que consideran importante el logro de los objetivos:

"...el que obtiene mejores resultados..."

"... aquel que entrega los conocimientos que tiene que entregar...”

“...tiene la capacidad de poder adecuar los objetivos de aprendizaje a las necesidades de los alumnos..."

Estos informantes logran incorporar un aspecto fundamental en la docencia, a saber, el hecho de que todos los estudiantes aprendan y desarrollen las competencias necesarias, y que éstas estén de acuerdo con las necesidades de los estudiantes, lo que implica conocerlos bien.

Para otros informantes, un buen docente es quien motiva e inquieta a sus estudiantes por el conocimiento y logra que todos ellos 
adquieran las competencias propuestas en los programas de estudios, tal es el caso de:

"...el que motiva a los alumnos a aprender más de la cuenta, él que logra que todos sus alumnos aprendan e interioricen ese aprendizaje..."

"...un gallo que inquiete a los alumnos, que motive, que sea motivador..."

"...que los alumnos se interesen, creo que para mí esa es la clave..."

Otro grupo de informantes aporta más características respecto de lo que es ser un buen docente. Además de poseer una experticia en la disciplina, debe poseer habilidades motivacionales y afectivas que favorezcan el proceso enseñanza-aprendizaje.

"... debe tener una base de conocimiento, experiencia en investigación, que sepa escuchar, que sea mitad psicólogo y mitad académico, y sobre todo valores..."

"...es una persona que tiene vocación, que le gusta enseñar, que se preocupa por investigar, por hacer más eficiente el proceso enseñanza-aprendizaje.

Este último grupo tiene una mirada más holística; considera aspectos tales como: la experticia de la disciplina que enseña, experiencia en investigación y vocación por la docencia.

En otra de las entrevistas el informante basa su reflexión en una película titulada "La Sociedad de los Poetas Muertos", que narra la historia de un profesor que logra motivar y despertar en sus estudiantes la pasión por la literatura y la poesía, enseñándoles a pensar, reflexionar y ser críticos, puesto que ésta es la única forma de ser verdaderamente libres. En la película se muestra a un profesor muy cercano de sus estudiantes, siempre rodeado y admirado por ellos. Este último punto, el docente lo relaciona con su idea de hacer docencia.

“...relaciono la película con aspectos que apuntan a encantar a los estudiantes, a que asistan con gusto 
a clases... que asistan a clases con la necesidad de aprender..."

El docente debe estar siempre consciente de su actuar docente y de cómo se relaciona con sus estudiantes, tratando de ser siempre un guía y un consejero, intentando practicar un estilo distinto de docencia más cercano y estimulador.

4.2.4. Categoría: el plan de estudios y los contenidos de enseñanza Con respecto a esta categoría, se encuentran dos tendencias en ambos grupos de estudio. Hay informantes que creen que hay contenidos de mayor relevancia que otros y que la teoría tiene gran importancia en el proceso de enseñanza, tal como lo demuestran las siguientes citas, tomadas de la entrevista semiestructurada:

"Yo siempre digo que la metodología de caso es la más importante..."

“...sí, hay temas más importantes, bases teóricas o conceptuales..."

"...el alumno tiene que saber muchos contenidos audiovisuales..."

Tanto para algunos informantes del área de Trabajo Social como para otros de Periodismo existen contenidos más importantes que otros en los planes de estudios, coincidiendo en que las asignaturas que ellos imparten son las de mayor importancia y, dentro de estas asignaturas, algunos contenidos por sobre otros, dando prioridad a la teoría por sobre la práctica.

Dentro de la misma categoría se encuentra otro grupo de docentes que tienen una mirada distinta del plan de estudios y de los contenidos que allí presentan, declarando que todos los contenidos son importantes, como lo demuestran los siguientes fragmentos:

"...todos los contenidos que están considerados dentro del programa son fundamentales..."

“...creo que son todos importantes..." 
"...no siento que una asignatura tenga mayor importancia que otra..."

\subsubsection{Categoría: la metodología de enseñanza}

Respecto de la metodología de enseñanza o los métodos utilizados por los informantes para llevar exitosamente la transposición didáctica, entendida como el hacer enseñable el saber disciplinario, destaca el trabajo en equipo, los trabajos prácticos y las clases expositivas cómo es el caso de los siguientes informantes:

"...explicar conceptos, definiciones, casos y la discusión... son metodologías que utilizo".

“...en las asignaturas teóricas no hay mucha opción metodológica; yo estoy ahí y trato de explicarles la materia de la forma más clara para ellos..."

"...bueno, voy mezclando clases, hay fases expositivas y prácticas, y hay otras que son mixtas..."

Algunos entrevistados señalan que el componente teórico tiene una fuerte presencia en sus asignaturas, lo que se traduce en clases que son fundamentalmente teóricas y, por ende, con énfasis en el rol del docente en el aula. Un caso distinto dentro de la misma categoría lo constituyen los informantes de la carrera de Periodismo que, por las capacidades que intentan desarrollar en sus estudiantes, presentan clases más prácticas, en las cuales es necesario que el estudiante elabore un producto final:

"...es el método práctico fundamentalmente..."

La mayoría de los entrevistados declara que sus metodologías son "prácticas", debido a que el estudiante debe poner inmediatamente en uso lo que está aprendiendo. En términos pedagógicos, esto se denomina "praxis": momento en el cual una teoría se convierte en parte de la experiencia del estudiante. En otras palabras, las teorías no son solamente absorbidas intelectual, sino son experimentadas en el mundo real y luego seguidas de una contemplación reflexiva. De este modo, los conceptos se conectan con la experiencia y el aprendizaje se transforma en significativo. 
"...mucha dinámica en clases, mucho trabajo
práctico".

Como se puede inferir, sus asignaturas tienen un fuerte componente práctico en donde sus estudiantes deben presentar resultados concretos, productos finales, más que demostrar sólo la adquisición de conocimientos.

Dentro de la misma categoría, el grupo de informantes de la carrera de Trabajo Social también declara tener metodologías mixtas, que combinan las clases expositivas, las lecturas, los trabajos grupales y la discusión, tal como se observa en los siguientes fragmentos:

"...utilizo harto trabajo en equipo, harto trabajo participativo..."

“...utilizo exposiciones, apoyo audiovisual, lectura, trabajos grupales y discusión...”

“...los trabajos grupales, lecturas colectivas... presentaciones grupales son mis metodologías preferidas..."

Como se puede observar en este grupo, quienes trabajan con un fuerte componente teórico utilizan el trabajo en equipo y la discusión como metodologías de enseñanza, con la idea de formar un profesional reflexivo que, además, sea capaz de integrarse fácilmente a un grupo de trabajo multidisciplinario.

\subsubsection{Categoría: la evaluación de los aprendizajes}

La mayoría de los informantes encuestados considera la evaluación como un proceso de verificación de la adquisición de los contenidos entregados durante el proceso enseñanza-aprendizaje, tal como lo demuestran las siguientes citas:

"...es la manera de comprobar que ciertos contenidos se hayan interiorizado en los alumnos..."

“...según yo, es corrección...”

"...sirve para saber si los alumnos han desarrollado el aprendizaje..." 
"...es la forma que yo tengo para saber si los chiquillos adquirieron los conocimientos o no..."

"...sirve para poder constatar los niveles de aprendizaje de los estudiantes..."

Los informantes citados sólo utilizan la evaluación al final de un periodo específico, como una herramienta para comprobar cuánto han logrado aprender sus estudiantes. También declaran utilizar sólo instrumentos evaluativos tradicionales, tales como: certámenes, tests, controles de lectura. Por lo general, consideran dos certámenes y el examen final en su proceso didáctico. Así lo demuestran las siguientes citas:

"...utilizo los certámenes, tests, los trabajos de investigación, informes de aplicación...”

“... hago certámenes y varios ejercicios prácticos..."

“...realizo dos certámenes, un examen, tres o cuatro evaluaciones complementarias, que son controles de lecturas..."

Algunos informantes de Periodismo tienen una visión similar a los de Trabajo Social, al ver la evaluación como una herramienta de medición; sin embargo, en el área de Periodismo algunos informantes buscan medir las competencias de los estudiantes mediante productos finales. El fundamento de medir las competencias por medio de actividades prácticas está en las asignaturas que estos docentes enseñan, puesto que ellas están asociadas al uso de las nuevas tecnologías de la información y comunicación. Determinados informantes declaran que el único instrumento evaluativo que ellos pueden utilizar para medir las competencias de sus estudiantes es el trabajo práctico, puesto que son el único modo de constatar si los estudiantes realmente desarrollaron las competencias propuestas al inicio del programa.

“...siempre pido un producto final...”

“...utilizo trabajos prácticos, creo que sólo aplico una prueba teórica en el año..." 
“...les hago ejercicios prácticos..."

4.2.7. Categoría: la actuación docente, el discurso y el impacto que provoca en los estudiantes

En relación con esta categoría, se evidencia el poder transformador que puede tener el discurso docente en la formación de nuevos profesionales. Esto se evidencia en los siguientes testimonios:

"...lo atractivo de esto es precisamente el desafío por construir una base sólida y consistente, que sea posible de transmitir y compartir con ellos, para que se muevan y se motiven por el desarrollo de la profesión y de las personas que son y serán..."

“...los profesores o quienes cumplen la labor docente, se constituyen en un referente para los alumnos y pueden impactar positivamente o negativamente en la formación de los estudiantes, más allá de los contenidos pedagógicos estrictos..."

Los informantes también reflexionan sobre la importancia de innovar en la práctica del proceso enseñanza-aprendizaje, mediante un enfoque más horizontal en la relación docente y discente, así lo demuestra la siguiente cita:

“... la película "La Sociedad de los Poetas Muertos" deja en evidencia como un profesor puede enfrentar el proceso enseñanza-aprendizaje de una manera innovadora, abierta, tolerante y profundamente provocativa, generando impactos significativos en el aprendizaje y en la vida de sus alumnos..."

\section{Conclusiones}

Los análisis presentados anteriormente han sido abordados fundamentalmente desde el punto de vista de la tríada didáctica que, en su esencia, reconoce tres variables del proceso enseñanzaaprendizaje: docente, discente y contenidos. La perspectiva cualitativa y, particularmente, la adscripción a la metodología de estudio de casos de esta investigación no permite realizar generalizaciones. Las conclusiones establecidas en esta sección deben entenderse en este contexto. 
Aunque los entrevistados de Trabajo Social y Periodismo provienen de áreas disciplinarias que no consideran formación pedagógica para sus estudiantes durante su paso por la universidad, resulta interesante constatar que ciertos informantes conceptualizan el aprendizaje como un proceso de interacción constante entre los actores involucrados en dicho proceso.

En ambas áreas se puede observar que la identidad profesional de los docentes de este estudio se ha formado por una serie de eventos con significado especial para los académicos, a saber:

- La formación de pre y posgrado.

- Las actividades de ayudantía en docencia y la dictación de la primera cátedra.

- La transición desde profesores a honorarios a profesores de planta de la institución.

- La primera experiencia docente con estudiantes, las interacciones y el reconocimiento de los estudiantes.

- Las conversaciones y consejos de pares, de docentes más experimentados y opiniones de ex alumnos.

- La motivación que logran con sus estudiantes y el grado de satisfacción que ello conlleva.

- La entrega de las primeras evaluaciones a los estudiantes.

- La retroalimentación de la primera evaluación docente.

- Los logros académicos y profesionales.

- Los profesores de la enseñanza media, quienes planteaban una relación pedagógica distinta que iba más allá de los contenidos.

- Los profesores de pre y posgrado.

- La formación de posgrado en lo disciplinar.

Gran parte de las creencias pedagógicas de estos docentes respecto a cómo se debe enseñar y aprender ya están asentadas en su mundo cognitivo antes de ingresar a la universidad, y están constituidas por hechos personales y académicos que los han marcado, de una u otra manera.

Tanto los docentes de enseñanza media, pregrado y posgrado como los pares tienen un fuerte impacto en la construcción de las 
creencias pedagógicas de estos informantes. Llama la atención la gran cantidad de creencias relacionadas con el rol del profesor en el proceso de enseñanza-aprendizaje. Las siguientes aparecen con mucha frecuencia.

Según los informantes de este estudio, el docente debería:

a) ser un agente de cambio, guiar a sus estudiantes, conocerlos;

b) tener altas expectativas de sus estudiantes, encantarlos y comprender su universo;

c) despertar admiración en los estudiantes;

d) utilizar metodologías didácticas innovadoras que motiven a sus estudiantes a aprobar el curso;

e) enseñar a sus estudiantes a reflexionar, pensar y ser críticos para ser verdaderamente libres;

f) establecer buenas relaciones de convivencia con los estudiantes en beneficio de la entrega de contenidos y teorías;

g) guiar a sus estudiantes;

h) no cometer los mismos errores que cometieron sus malos profesores;

i) tener un estilo distinto de docencia: cercano y entusiasta;

j) integrar sabiduría, conocimiento, humildad, carisma, elocuencia, moral y amor;

k) mostrar agrado y disposición en la acción docente, y

1) entregar el sustento teórico y las herramientas necesarias para que los estudiantes puedan desarrollarse como profesionales.

Todas las funciones descritas y que un docente debería realizar en el proceso didáctico se matizan con otras creencias de los académicos respecto del proceso enseñanza-aprendizaje:

- El conocimiento y la técnica son más importantes que las herramientas.

- El dominio de un área temática de su especialidad y la metodología didáctica para enseñarla.

- El compromiso paulatino que se desarrolla con la labor docente.

- El desarrollo de una práctica más horizontal con los estudiantes.

- El conocimiento previo de los estudiantes y de cómo éstos aprenden. 
- Los estudiantes deben estar dispuestos a aceptar la ayuda del docente para así desarrollar nuevas capacidades.

- El conocimiento entrega libertad cuando los estudiantes son capaces de pensar y actuar por sí solos.

- La responsabilidad profesional de un docente es fundamental al transmitir sus conocimientos a los estudiantes.

- El discurso académico tiene un poder transformador.

- La innovación en la práctica docente es fundamental.

La proveniencia de los informantes del área humanista se observa nítidamente desde el momento que indican que el estudiante de enseñanza superior que desea estudiar Periodismo o Trabajo Social debe poseer habilidades sociales, analíticas y comunicativas que le permitan desempeñarse eficientemente en el mercado laboral.

Por otro lado, la gran mayoría de los informantes considera que sus estudiantes no saben cómo aprender y utilizan la memorización como estrategia principal de aprendizaje. Evidencian las pobres habilidades de comprensión de lectura de los estudiantes y, a su vez, corroboran el hecho de que el estudiante aprende haciendo.

El logro de aprendizajes de calidad por parte de los estudiantes requiere de un esfuerzo particular por parte del profesor. En este sentido, para los informantes, un buen docente es el que motiva, inquieta e interesa a los estudiantes. También, aquel que es capaz de obtener los mejores resultados, adecuar los objetivos de aprendizaje y escuchar a los estudiantes. En definitiva, debería poseer una base de conocimiento y experiencia investigativa que puede aplicar en su práctica pedagógica.

Finalmente, y con las limitaciones que presenta este estudio, el mejoramiento de los procesos de enseñanza-aprendizaje debería suponer un cambio en las creencias de uno de los actores principales del proceso educativo: el docente. Desde la cognición, este grupo interpreta y adapta las innovaciones pedagógicas y promueve u obstaculiza el aprendizaje de sus estudiantes. El sistema de creencias de los profesores -como constructo personal, complejo y subjetivo- podría tener un impacto considerable tanto en sus percepciones y juicios como en su comportamiento en el aula. 
En la situación particular del proceso enseñanza-aprendizaje, el entramado intelectual del docente se originaría y desarrollaría en la experiencia. Las creencias serían particulares y específicas y estarían determinadas por los contextos educativos y profesionales donde el docente se ha desenvuelto. El proceso de exploración de la cognición docente puede ayudar a enriquecer la conceptualización sobre el proceso de enseñanza-aprendizaje $y$, además, sirve como base para la autoevaluación y reflexión docente, componentes importantes del desarrollo profesional en la educación superior.

Este artículo se inserta en el contexto del proyecto FONDECYT 1060622 "El sistema de creencias y prácticas pedagógicas del docente universitario y su implicancia en el proceso de enseñanza-aprendizaje", realizado entre 2006 y 2008. Los datos fueron acopiados por Claudio Puentes M., alumno del Programa de Posgrado de Ciencias de la Educación, Universidad Católica de la Santísima Concepción, en el contexto de su tesis de magíster (supervisada por Claudio Díaz).

\section{Referencias bibliográficas}

Andrés, G. y Echeverri, P. (2001) Pensamiento docente y práctica pedagógica. Una investigación sobre el pensamiento práctico de los docentes. Bogotá: Magisterio.

Andrews, S. (2003) Just like instant noodles': L2 teachers and their beliefs about grammar pedagogy. Teachers and Thinking: Theory and Practice, 9, pp. 351-370.

Appel, J. (1995) Diary of a language teacher. Oxford: Heinemann.

Bailey K., Curtis, A. \& Nunan, D. (2001) Pursuing professional development. Boston: Newbury House. Teacher Development.

Ben-Peretz, M. (2002) Retired teachers reflect on learning from experience. Teachers and Thinking: Theory and Practice, 8, pp. 312-324.

Biddle, B., Good, T. y Goodson, I. (2000) La enseñanza y los profesores I. La profesión de enseñar. Buenos Aires: Paidós.

Borg, S. (2003) Teacher cognition in language teaching: a review of research on what language teachers think, know, believe, and do. The International Abstracting. Journal for Language Teachers and Applied Linguists, 36, pp. 81-109. 
Cárdenas, A., Rodríguez, A. y Torres, R. (2000) El maestro protagonista del cambio educativo. Bogotá: Magisterio.

Camps, A. (2001) El aula como espacio de investigación y reflexión. Investigaciones en didáctica de la lengua. Barcelona: Graó.

Carusetta, E. \& Cranton, P. (2005) Nurturing authenticity: a conversation with teachers. Teaching in Higher Education, 10, pp. 285-297.

Crookes, G. (2003) A practicum in Tesol. Professional development through teaching practice. Cambridge: Cambridge Language Education.

Frabboni, F. (2001) El libro de la pedagogía y la didáctica. I: La educación. Madrid: Editorial Popular.

Freeman, D. (2002) The hidden side of the work: Teacher knowledge and learning to teach. A perspective from North American educational research on teacher education in English language teaching. Language Teaching. The International Abstracting Journal for Language Teachers and Applied Linguists, 35, pp. 1-13.

Gelhard, J. \& Oprandy, R. (1999) Language teaching awareness. A guide to exploring beliefs and practices. Cambridge: Cambridge University Press.

González S., Río, E. y Rosales, S. (2001) El Currículum oculto en la escuela. Buenos Aires: Lumen Humanitas.

Goodson, I. \& Numan, U. (2002) Teacher's life worlds, agency and policy contexts. Teachers and Thinking: Theory and Practice, 8, pp. 269-277.

Hamel, F. (2003) Teacher understanding of student understanding: Revising the gap between teacher conceptions and students' ways with literature. Research in the Teaching of English, 38, pp. 49-84.

Hashweh, M. (2005) Teacher pedagogical constructions: a reconfiguration of pedagogical content knowledge. Teachers and Thinking: Theory and Practice, 11, pp. 273-292.

Johnson, K. \& Golombek, P. (2002) Teachers' narrative inquiry as professional development. Cambridge: Cambridge University Press.

Johnstone, R. (2000) Research on language learning and teaching: 1999. Language Teaching. The International Abstracting Journal for Language Teachers and Applied Linguists, 33, pp. 141-162.

Kansanen, P., Tirri, K., Meri, et al. (2000) Teachers' pedagogical thinking. New York: American University Studies.

Libedinsky, M. (2001) La innovación en la enseñanza. Buenos Aires: Paidós. 
Menin, O. (2001) Pedagogía y Universidad. Currículo, didáctica y evaluación. Santa Fe: Ediciones Homo sapiens.

Muchmore, J. (2004) A teachers' life. San Francisco: Backalong books.

Richard-Amato, P. (2003) Making it happen. New York: Longman.

Richards, J. \& Nunan D. (1990) Second Language Teacher Education. CUP.

Sandín, M. Paz (2003) Investigación cualitativa en educación. Fundamentos y tradiciones. Madrid: McGraw Hill.

Sanjurjo, L. (2002) La formación práctica de los docentes. Reflexión y acción en el aula. Santa Fe: Homo sapiens.

Sevillano, M. L. (2007) Investigar para innovar en enseñanza. Madrid: Pearson.

Tsui, A. (2003) Understanding expertise in teaching. Cambridge: Cambridge University Press.

Undurraga, C. (2007) ¿Cómo aprenden los adultos? Santiago de Chile: Ediciones Pontificia Universidad Católica de Chile.

Woods, D. (1996) Teacher cognition in language teaching: beliefs, decision-making and classroom practice. Cambridge: Cambridge University Press.

Recibido: 26 agosto de 2008

Aceptado: 4 noviembre de 2008 